\title{
NON-LINEAR RESONANCES IN ACCRETION DISKS AND QPOs
}

\author{
Włodek Kluźniak ${ }^{1,2}$ \\ RESUMEN
}

Secundamos las oscilaciones no-lineales en el disco de acreción como una explicación de las "oscilaciones cuasiperiódicas" de alta frecuencia observadas en las curvas de luz de las binarias de rayos X de baja masa que contienen estrellas de neutrones, agujeros negros o enanas blancas.

\section{ABSTRACT}

Non-linear oscillations in the accretion disk are favored as an explanation of high-frequency QPOs observed in the light curves of low-mass X-ray binaries containing neutron stars, black holes, or white dwarfs.

\section{Kiy Words: BLACK HOLE PHYSICS - STARS: NEUTRON - X-RAYS: BINARIES}

\section{QPOs}

Several variable frequencies have been detected in the Fourier spectra of the light curves of several semi-detached binaries, where the accreting compact object is either a white dwarf, or a neutron star, or a black hole. The width of the Fourier peaks clearly shows that these "quasi-periodic oscillations" are not coherent, and the time-scale of variability also indicates that none of these frequencies can be attributed to the rotation rate of the compact stellar remnant. Some of the frequencies are correlated (Psaltis, Belloni, van der Klis, 1999). That QPOs in neutron stars, black holes and white dwarfs all follow the same correlation (Mauche 2002, Warner, Woudt \& Pretorius 2003) indicates that the same phenomenon is observed in all three types of sources. This points to the only structure common to all of these systems, the accretion disk, as the site where the frequencies originate. QPOs have been reviewed recently in van der Klis (2000) and Warner (2003).

The highest-frequency QPOs (HF QPOs) attract most attention, For one thing, a QPO at $1.2 \mathrm{kHz}$ has twice the frequency of the fastest known radio pulsar. For another, they seem to be occurring at the dynamical frequency close to the inner radius of the disk: $\mathrm{kHz}$ for neutron stars, $\mathrm{hHz}$ for black holes, and fractions of a $\mathrm{Hz}$ for white dwarfs. No harmonics of these frequencies have ever been detected. These HF QPOs in neutron stars and black holes occur in pairs of characteristic frequencies, unlike their single counterparts in white dwarfs. Could this difference be ascribed to general relativity?

\footnotetext{
${ }^{1}$ Institute of Astronomy, Zielona Góra University

${ }^{2}$ Copernicus Astronomical Center.
}

\section{TWO CHARACTERISTIC FREQUENCIES}

The old suggestion that one frequency is an orbital frequency and the other is the beat of the first with the constant stellar spin frequency is untenable, because the frequency difference of the two QPOS varies by too much. An interesting question to ask is what else can give rise to two variable, but characteristic frequencies. The only answer suggested to date is that the two frequencies are a result of nonlinear resonance (Kluźniak and Abramowicz 2000, 2003, and references therein).

\section{NON-LINEAR RESONANCE}

So far, this suggestion seems to work well. McClintock \& Remillard (2003) have found that HF QPOs in microquasar occur at twin frequencies in a 2:3 ratio, as expected for non-linear resonance (Abramowicz \& Kluźniak 2003). And in the accreting, transient, millisecond pulsar, Wijnands et al. (2003) find two frequencies separated by onehalf of the $401 \mathrm{~Hz}$ spin frequency-such subharmonic separation is a hallmark of non-linear resonance (Kluźniak et al., 2003; Lee, Abramowicz \& Kluźniak 2004; Kluźniak, Abramowicz \& Lee 2004). The frequency-frequency correlation of the two $\mathrm{HF}$ QPOs in Sco X-1 (van der Klis et al. 1997), can be explained by a system slightly off resonance, near parametric resonance between vertical and radial epicyclic frequencies (Abramowicz et al. 2003; Rebusco 2004).

More work must be done, in particular to explain the high quality $(Q>100)$ of the oscillation, but it seems to be very difficult to avoid non-linear coupling of accretion-disk modes as the explanation of HF QPOs in neutron stars and black holes.

Supported in part by KBN grant 2P03D01424. 


\section{REFERENCES}

Abramowicz M.A. \& Kluźniak W. 2003, in X-Ray Timing 2003: Rossi and Beyond, P. Kaaret, J. Swank, eds. in press, astro-ph/0312396

Abramowicz M.A., Karas V., Kluźniak W. Lee W., Rebusco P. 2003 Publ. Astr. Soc. Japan., 55, 467

Kluźniak W., Abramowicz M.A. 2000, Phys. Rev. Lett. (submitted), astro-ph/0105057

Kluźniak W., Abramowicz M.A. 2003, 12th Workshop on General Relativity and Gravitation, (Tokyo: Tokyo University Press), astro-ph/0304345

Kluźniak W., Abramowicz M.A., Kato, S., Lee, W., Stergioulas, N. 2003, ApJL in press, astro-ph/0308035

Kluźniak W., Abramowicz M.A., Lee, W. 2004, in X-Ray Timing 2003: Rossi and Beyond, P. Kaaret, J. Swank, eds in press, astro-ph/0402013
Lee, W.H., Abramowicz M.A., Kluźniak, W. 2004. ApJLett. in press

Mauche, C. 2002, ApJ 580, 423

McClintock J.E, Remillard R.A. 2003, astro-ph/0306213 v. 2

Psaltis D., Belloni T., van der Klis .I. 1999, ApJ 520. 262

Rebusco, P. 2004, submitted

van der Klis M., Wijnands, R.A.D., Horne, K., Chen. W 1997, ApJ 481, L97

van der Klis M. 2000, Ann. Rev. of A\&A 38, 717

Warner, B., astro-ph/0312182

Warner, B., Woudt, P.A., Pretorius, M.L. 2003, MNRAS 344,1193

Wijnands, R., van der Klis, M., Homan, J., Chakrabarty, D., Markwardt, C. B., Morgan, F. H. 2003 Nature, 6944,44

Włodek Kluźniak, Institute of Astronomy, Zielona Góra University, ul. Lubuska 2, 65-265 Zielona Góra. Poland. (wlodek@camk.edu.pl). 